Rev. Elev. Med. vét. Pays trop., 1975, 28 (4) : 463-472

\title{
Valeur de la méthode d'immunofluorescence indirecte dans le diagnostic des trypanosomiases bovines et leur étude épizootiologique
}

\author{
par S. M. TOURE (*), M. SEYDI (**), M. SEYE (*) et B. KEBE (*)
}

\section{RESUME}

Des épreuves d'immunofluorescence indirecte, pratiquées dans les Trypanosomiases bovines, il ressort que cette méthode, quoique très sensible et utile dans les études d'épizootologie et les recherches sur les anticorps, a cependant un intérêt plus limité en matière de diagnostic du fait de l'impossibilité de déceler des infections précoces, des incertitudes quant aux espèces de trypanosomes en cause et aussi parce que la présence d'anticorps ne traduit pas nécessairement une infection présente.

\section{INTRODUCTION}

La méthode d'immunofluorescence indirecte vient de s'ajouter, dans le diagnostic expérimental des trypanosomiases animales, à de nombreux autres tests sérologiques, de sensibilité et de spécificité variables selon les cas et qui ont eu un bonheur inégal suivant leurs utilisateurs. La conception de l'immunofluorescence remonte à plusieurs décennies déjà (4), mais ses applications en parasitologie, particulièrement dans le diagnostic expérimental des trypanosomiases, selon la méthode indirecte, peuvent être considérées comme relativement récentes : maladie de Chagas à Trypanosoma cruzi par FIFE et MUSCHEL, 1959 (6), trypanosomiases expérimentales ou naturelles dues à $T$. rhodesiense ou à $T$. gambiense, par SADUN et DUXBURY, 1963 (11) (12). D'autres applications pratiques, se rapportant cette fois aux trypansomiases des bovins, ont été réalisées ces dernières années par divers auteurs dont la plupart ont travaillé sur du bétail africain.

(*) Institut sénégalais de Recherches agricoles (I.S.R.A.) Laboratoire nat. de l'Elevage et de Recherches vétérinaires, B.P. 2057, Dakar, Sénégat:

(**) Institut de Technologie alimentaire, Dakar, Sénégal.
La présente note a pour objet de passer en revue les résultats que nous avons obtenus par la méthode d'immunofluorescence indirecte, telle qu'elle est pratiquée ici depuis 1972 à maintenant, dans le diagnostic et l'étude épizootiologique des trypanosomiases bovines au Sénégal, et aussi de faire une étude critique de cette méthode en nous appuyant sur nos observations et celles d'autres expérimentateurs.

\section{MATERIEL ET METHODES}

\section{Conjugués}

Le conjugué commercial anti-sérum que nous avons utilisé au cours des premières épreuves ne nous ayant pas donné complète satisfaction, nous l'avons abandonné au profit de conjugués préparés dans notre laboratoire comme suit : inoculation pendant trois semaines à des lapins de sérum de bœuf; récolte du sérum de ces lapins, isolement des globulines par le sulfate d'Ammonium en solution saturée 4,6 M. Les fractions anti globulines de bovin sont conjuguées à l'isothiocyanate de fluorescéine après dialyse contre un soluté physiologique tamponné et détermination de la concentration protéi- 
que au spectrophotomètre. La conjugaison est suivie d'épuisement à travers une colonne de Sephadex G 50, de concentration par le polyéthylène glycol P.M. 4000 et de purification par une poudre de foie.

Plusieurs lots de conjugués ont été préparés et, au moment de l'emploi, titrés et étalonnés. La diluion optimale pour les épreuves de fluorescence est comprise entre $1 / 10$ et $1 / 32$. En plus de ces conjugués antiglobulines de bœuf, il a été préparé des conjugués anti-IgM de bovins trypanosomés pour comparer la sensibilité des réactions obtenues avec ces conjugués et les précédents. Dans certaines épreuves, on a pratiqué la contre-coloration par le Bleu d'Evans.

\section{Antigènes}

Les antigènes proviennent d'animaux de laboratoire (rat ou souris) pour les souches de Trypanosoma brucei et $T$. congolense et de chèvre pour les souches de $T$. vivax. A titre de comparaison, on a utilisé en outre un antigène $T$. theileri de culture. Tous ces antigènes sont étalés sur lames et fixés à l'acétone.

\section{Sérums testés}

a) Infection expérimentale. - Cinq veaux ont été infectés par une souche de $T$. brucei et leurs sérums récoltés respectivement aux $15^{\mathrm{e}}$, $22^{\mathrm{e}}$ et $76^{\mathrm{e}}$ jours après infection; puis ces animaux sont traités au $76^{\mathrm{e}}$ jour par l'acéturate de Diminazène et saignés aux $15^{\mathrm{e}}, 65^{\mathrm{e}}, 106^{\mathrm{e}}$ et $145^{\mathrm{e}}$ jours après traitement à l'exception d'un veau mort avant le traitement). Cinq autres veaux ont été infectés par $T$. congolense; le sérum est récolté uniquement, au $33^{\mathrm{a}}$ jour après infection; puis les animaux sont traités (sauf un, mort avant traitement) et leurs sérums récoltés comme ci-dessus aux $15^{\mathrm{e}}$ et $65^{\mathrm{e}}$ jours pour les 4 animaux, aux $106^{\mathrm{e}}$ et $145^{\mathrm{e}}$ jours pour 2 d'entre eux.

Dans une épreuve plus récente, le sérum de deux boufs a été analysé $15 \mathrm{j}, 21 \mathrm{j}$ et $28 \mathrm{j}$ après infection par une souche de $T$. congolense qui était l'objet d'un test de virulence.

$\mathrm{Au}$ cours de ces épreuves, le sérum d'animaux témoins non infectés a été récolté parallèlement.

Tous les bovins d'expérience sont de race Zébu et ils ont été maintenus au laboratoire pendant plus d'un an pour les différentes expériences.

Pour chaque animal, on a procédé à une analyse parasitologique du sang: un des veaux infectés par $T$. brucei avait des microfilaires de Setaria sp. D'autres part, l'hémoculture a révélé la présence de $T$. theileri chez un des animaux infectés avec $T$. congolense.

b) Infection naturelle. - Les sérums testẻs ici proviennent de bovins de diverses régions du Sénégal, situées dans l'aire de distribution des glossines, mais aussi, à titre de comparaison, hors de cette zone. Chaque prélèvement de sérum est accompagné de frottis et gouttes épaisses colorées au Giemsa suivant la méthode standard de détection des trypanosomes. Dans certains cas, le sang des animaux a fait l'objet d'hémoculture pour déceler $T$. theileri, comme ci-dessus. Enfin dans quelques localités, on a procédé à des récoltes de sang sur papier filtre pour analyse d'immunofluorescence par la méthode des confettis selon CUNNINGHAM et al., (5).

\section{Epreuves et résultats}

Les étapes de la méthode indirecte d'immunofluorescence ont été codifiées dans de nombreuses publications et il n'y a pas lieu d'y revenir ici. Nous avons suivi les différentes phases, comme indiqué par AMBROISE-THOMAS, (1) (2), à qui revient le grand mérite d'avoir étudié par ce procédé dix parasitoses différentes, en analysant plus de 14000 sérums.

Les lectures sont pratiquées sur microscope Wild M 20 doté d'une base de fluorescence utilisant une lampe à vapeur de Mercure HBO 200. Un équipement de microphotographie permet de prendre des photographies en noir et blanc ou en couleurs; nous avons utilisé avec de bons résultats un film Ansco 200 ASA.

L'interprétation des résultats a souvent posé des problèmes difficiles du fait de la subjectivité liée à l'appréciation du degré de fluorescence. Dans les premières épreuves, celles de diagnostic individuel, nous avons adopté l'échelle proposée par WERY et al., (18) dans une étude de la maladie du sommeil :

- Trypanosomes à peine visible sur fond noir : $\mp$

- Trypanosomes visibles, mais sans vraie fluorescence : + 
- Trypanosomes contrastant sur fond noir et avec une pâle fluorescence: ++

- Trypanosomes présentant une fluorescence brillante sur fond noir : +++

- Trypanosomes présentant une fluorescence très brillante sur fond noir : ++++

Pour simplifier la représentation des résultats, cette échelle est chiffrée ici de zéro à 4 , suivant l'intensité croissante.

D'un point de vue pratique, seules les réactions de degré 3 et 4 sont considérées comme positives; celles de degré 2 sont douteuses et au-dessous elles sont négatives. Dans les tests d'épizootiologie, on s'est borné à sérier les cas en positifs et négatifs sans chiffrer l'intensité de la fluorescence.

Enfin, nous avons suivi Ambrois-Thomas (1) pour présenter certains résultats par le calcul statistique de la moyenne géométrique des titres d'anticorps (geometrical Mean Reciprocal Titer (G.M.R.T.) selon Waugh :

$$
\text { GMRT }=\operatorname{antilog} \Sigma \mathrm{f}(\log \mathrm{x}) / \mathrm{N}
$$

où $\mathrm{x}$ représente les titres réciproques d'anticorps, c'est-à-dire l'inverse des différents titres d'anticorps,

f le nombre de sérums qui ont respectivement donné chacun de ces titres,

$\mathrm{N}$ le nombre total de sérums.

Cela revient à prendre l'antilog de la valeur obtenue en divisant par le nombre total de sérums analysés, la somme des produits du logarithme de l'inverse de chaque titre d'anticorps par le nombre de sérums réagissant à ce titre.

\section{RESULTATS}

\section{Infections expérimentales}

1. Infection expérimentale par Trypanosoma brucei brucei

Dans ce premier lot, 5 veaux inoculés le 11 décembre 1972 avec une souche maintenue sur souris, n'ont présenté de parasitémie lisible que le 8 janvier suivant (4 positifs sur 5); les semaines suivantes, la parasitémie est irrégulière, toujours faible, à l'exception du $\mathrm{n}^{\circ} 55$ qui présente une forte infection les 15 et 16 janvier 1973 et meurt le 17 février, soit 67 jours après l'inoculation; les quatre autres animaux sont traités par l'acéturate de Dimina- zène le 26 février, soit 76 jours après l'inoculation. Les récoltes de sérum, opérées aux $15^{\mathrm{e}}$ et $22^{\mathrm{e}}$ jours qui suivent l'inoculation (parasitémie non décelée) ainsi qu'au $76^{\mathrm{e}}$ jour (parasitémie lisible) donnent les résultats présentés dans les tableaux qui suivent.

TABL. $\mathrm{N}^{\circ} \mathrm{I}$-Infection expërimentale à $T$. brucei, 15

jours après infection, antigène homologue.

\begin{tabular}{|c|c|c|c|c|c|}
\hline \multirow{2}{*}{\multicolumn{2}{|c|}{$\mathrm{N}^{\circ}$ des bovins }} & \multicolumn{4}{|c|}{ Dilutions des sërums } \\
\hline & & Pur & $1 / 10$ & $1 / 20$ & $1 / 40$ \\
\hline & 352 & 0 à 1 & 1 & 1 & 2 \\
\hline & 354 & 2 & 2 & 2 & 2 \\
\hline & 355 & 0 à 1 & 1 & 0 à 1 & 0 à 1 \\
\hline & 356 & 0 à 1 & 2 & 1 & 1 \\
\hline & 358 & 2 & 2 & 2 & 2 \\
\hline Témoin négatif & 359 & 0 & 0 & 0 & 0 \\
\hline \multicolumn{2}{|l|}{ Conjugué seu1 } & \multicolumn{4}{|c|}{ Fluorescence nulle } \\
\hline
\end{tabular}

A l'absence de trypanosomes sur les frottis et gouttes épaisses, correspondent des réactions négatives de fluorescence, même à faible dilution. A noter cependant que le conjugué utilisé est une préparation commerciale dont les conditions de conservation ont pu altérer la qualité.

TABL. $\mathrm{N}^{*}$ II-Infection expérimentale à T.bmucei, 22 et 76 jours après infection, antigène homologue.

\begin{tabular}{|c|c|c|c|c|c|c|c|c|}
\hline \multirow{2}{*}{\multicolumn{2}{|c|}{ Nos bovins }} & \multicolumn{3}{|c|}{22 jours après } & \multicolumn{4}{|c|}{76 jours après } \\
\hline & & $1 / 10$ & $1 / 20$ & $1 / 40$ & $1 / 40$ & $1 / 80$ & $1 / 160$ & $1 / 320$ \\
\hline \multirow{5}{*}{ Infectés } & 352 & 2 & 2 & 2 & - & - & - & - \\
\hline & 354 & 3 & 3 & 2 & 4 & 4 & 4 & 2 \\
\hline & 355 & 2 & 2 & 2 & $4^{+}$ & $4^{+}$ & $3^{+}$ & $3^{+}$ \\
\hline & 356 & 1 & 1 & 2 & 3 & 3 & 3 & 2 \\
\hline & 358 & 1 & 1 & 1 & 3 & 3 & 3 & 2 \\
\hline \multirow{3}{*}{$\begin{array}{l}\text { Tëmoins } \\
\text { négatifs }\end{array}$} & 359 & 1 & 0 & 0 & - & - & - & - \\
\hline & 353 & - & - & - & 1 & 1 & 1 & 0 \\
\hline & 284 & - & - & - & 1 & 1 & 0 & 0 \\
\hline \multicolumn{2}{|c|}{ Conjugué seul } & \multicolumn{7}{|c|}{ Fluorescence nulle } \\
\hline
\end{tabular}

Dans ces deux premiers tableaux, on note qu'à mesure que l'infection se prolonge, les réactions sont positives à des dilutions de plus en plus élevées. Le sérum du bovin $\mathrm{n}^{\circ} 355$ correspond au $67^{\mathrm{e}}$ jour (+). Quelques uns de ces sérums ont été testés contre un antigène $T$. congolense mais les résultats sont peu nets, 
même avec des sérums qui réagissent à dilution élevée sur l'antigène $T$. brucei.

TABL, $N^{\circ}$ III-Comparaison des rësultats du tableau $\mathrm{n}{ }^{\circ} I I$

\begin{tabular}{|c|c|c|c|c|}
\hline Dates & Titres & $\begin{array}{l}\text { Sérum } \\
\text { rêagissant }\end{array}$ & $\begin{array}{l}\text { Total } \\
\text { analýsé }\end{array}$ & GMRT \\
\hline $22 \mathrm{e} \mathrm{j}$. & $\begin{array}{l}\text { négatif } \\
1 / 10 \\
1 / 20\end{array}$ & $\begin{array}{l}4 \\
0 \\
1\end{array}$ & 5 & 1,8 \\
\hline $76 \mathrm{e} \mathrm{j.}$ & $\begin{array}{l}\text { régatif } \\
1 / 40 \\
1 / 80 \\
1 / 160 \\
1 / 320\end{array}$ & $\begin{array}{l}0 \\
0 \\
0 \\
3 \\
1\end{array}$ & 4 & 190,2 \\
\hline
\end{tabular}

2. Infection expérimentale par Trypanosoma congolense

Un second lot de 5 veaux a été inoculé le 23 janvier 1973 avec une souche de $T$. congolense, maintenue sur souris. La parasitémie est lisible une semaine après, et le 3 février tous les animaux présentent un très grand nombre de trypanosomes dans le sang. Ils restent fortement parasités jusqu'à leur traitement le 26 février (soit la même date que les animaux du premier lot). Un des animaux est mort le jour où a été prise la décision de traitement, soit 34 jours après l'inoculation, et les autres ont fait l'objet de récolte de sérum.

Les sérums du $34^{e}$ jour ne réagissent que faiblement (fluorescence 2 à $1 / 80$ et 1 à $1 / 160$ ) avec un antigène hétérologue $T$. brucei et encore plus faiblement avec un antigène homolo- gue. Les mêmes faits ont été constatés avec des sérums récoltés 15,21 et 28 jours après infection de deux zébus soumis à un test de virulence de $T$. congolense. L'antigène $T$. brucei nous a semblé meilleur, que l'infection soit due à $T$. congolense ou à $T$. brucei.

\section{Persistance des anticorps après traitement des 2 lots}

Les 2 lots d'animaux, traités le même jour, ont fourni des sérums analysés aux $15^{\mathrm{e}}, 65^{\mathrm{e}}$ $106^{\mathrm{e}}$ et $145^{\mathrm{e}}$ jour qui suivent le traitement. Les résultats obtenus sont résumés dans le tableau $n^{0}$ IV.

Les réactions obtenues avec les sérums des animaux à $T$. congolense sont sensiblement de même intensité qu'avant le traitement: elles restent faibles. Quant aux animaux antérieurement infectés par $T$. brucei, ils continuent à héberger des anticorps qui persistent à un niveau élevé jusqu'au $145^{\mathrm{e}}$ jour, comme l'indique d'autre part la G.M.R.T. qui reste égale à 160 dans les quatre périodes du tableau.

\section{Réinfections suivies de traitement}

Un an après le début de l'expérimentation, certains animaux ont été réinfectés : le $\mathbf{n}^{\circ} 365$ par T. brucei (14 janvier 1974) le $\mathrm{n}^{\circ} 354$ par par T. vivax (le 24 janvier 1974). Tous ces bovins ont été traités le 4 mars 1974 alors qu'ils présentaient une parasitémie marquée. Ensuite, les sérums, récoltés tous les 15 jours ont donné les résultats suivants avec un antigène $T$. brucei $i$ (tableau $\mathrm{n}^{\circ} \mathrm{V}$ ).

TABL. $N^{\circ}$ IV-Persistance des anticorps aprẽs traitement : comparaison entre les deux lots d'aprēs 1 'êchelle de fluorescence.

\begin{tabular}{|c|c|c|c|c|c|c|c|c|c|c|c|}
\hline \multirow{2}{*}{$\mathrm{N}^{\circ}$ des bovins } & & \multirow{2}{*}{$\begin{array}{l}\text { Infection } \\
\text { initiale }\end{array}$} & \multirow{2}{*}{ Antigène } & \multicolumn{2}{|c|}{$15 \mathrm{j}$. après } & \multicolumn{2}{|c|}{$65 \mathrm{j}$. après } & \multicolumn{2}{|c|}{$106 \mathrm{j}$. après } & \multicolumn{2}{|c|}{$145 \mathrm{j}$. après } \\
\hline & & & & $1 / 80$ & $1 / 160$ & $1 / 80$ & $1 / 160$ & $1 / 80$ & $1 / 160$ & $1 / 80$ & $1 / 160$ \\
\hline \multirow{7}{*}{$\begin{array}{l}\text { Initialement } \\
\text { infectës }\end{array}$} & 354 & T. brucei & T. brucei & 3 & 3 & 3 & 3 & 3 & - & 3 & 3 \\
\hline & 356 & T. bruce: & T. brucei & 3 & 3 & 3 & 3 & 3 & - & - & - \\
\hline & 358 & $\vec{T}$. bruaei & T. brucei & 3 & 3 & 3 & 3 & 3 & - & - & - \\
\hline & 285 & T. congolense & 7. bracei & 2 & 2 & 2 & 1 & - & - & - & - \\
\hline & 351 & T. oongolense & T. brucei & 2 & 2 & 2 & 2 & 1 & - & (2)? & 1 \\
\hline & 359 & I. congctense & T. brucei & 1 & 1 & 1 & 1 & - & - & - & - \\
\hline & 365 & T. congclense & T. brucei & 2 & 1 & 1 & 0 & 1 & - & (2)? & 1 \\
\hline \multirow{3}{*}{ Témoins } & 353 & & T. brucei & 1 & 0 & 1 & 0 & - & - & - & - \\
\hline & 284 & & T. bmaei & 0 & 0 & 0 & 1 & - & - & - & - \\
\hline & 289 & & T. bruati & 0 & 0 & 1 & 0 & 0 & - & 0 & 0 \\
\hline Conjugué seul & & & T. bmuce & \multicolumn{8}{|c|}{ Fluorescence nulle } \\
\hline
\end{tabular}


TABL. $N^{\circ} \mathrm{V}$-Degré de fluorescence dans les rểnfections suivies de traitement.

\begin{tabular}{|c|c|c|c|c|c|c|c|c|}
\hline \multirow{2}{*}{$\begin{array}{l}\mathrm{N}^{\circ} \text { des } \\
\text { bovins }\end{array}$} & \multirow{2}{*}{$\begin{array}{l}\text { Infection } \\
\text { initiale }\end{array}$} & \multirow{2}{*}{$\begin{array}{l}\text { Seconde } \\
\text { infection }\end{array}$} & \multicolumn{2}{|c|}{ Avant traitement } & \multicolumn{4}{|c|}{ Après traitement } \\
\hline & & & Durée & Réaction & 15 jours & 30 jours & 45 jours & 60 jours \\
\hline 365 & I. congotense & T. brucei & 49 jours & 4 & 4 & 3 & 3 & 3 \\
\hline 354 & T. órucet & I. vivax & 39 & 3 & 3 & 3 & 3 & 1 \\
\hline 357 & T. congcience & T. congolense & & 3 & 3 & 2 & 1 & 1 \\
\hline 359 & T. congotance & T. congolense & 38 & 3 & 3 & 1 & 1 & 1 \\
\hline
\end{tabular}

Les observations ci-dessus ne portent que sur quelques animaux mais permettent, en première approche, de faire quelques comparaisons. Les animaux initialement infectés, puis réinfectés par $T$. congolense ont une production d'anticorps plus élevée qu'au cours de leur première infection, après une évolution de la maladié en un nombre de jours voisins ( 34 jours et 39 jours), mais il y a pareillement une baisse assez rapide du niveau des anticorps. Lorsque l'infection initiale est due à $T$.congolense et la consécutive à $T$. brucei, le niveau des anticorps est très élevé et semble se maintenir assez longtemps, comme on a pu le constater précédemment. L'intensité des réactions hétérologues observées avec $T$, vivax est assez forte et la persistance des anticorps plus longue que dans l'infection due à $T$. congolense mais plus faible que dans celle due à $T$. brucei.

A la lumière des observations résumées dans les tableaux $\mathrm{n}^{\circ} \mathrm{IV}$ et $\mathrm{n}^{\circ} \mathrm{V}$, on ne saurait préjuger de la durée réelle de persistance des anticorps mais un fait est certain qui est de la plus grande importance en matière de diagnostic individuel, c'est que les réactions d'immunofluorescence sont positives avec les sérums d'animaux antérieurement infectés depuis plusieurs semaines mais guéris ensuite tant sur le plan clinique que sur le plan parasitologique, grâce à un traitement trypanocide; les réactions restent positives, selon les espèces de trypanosomes, plusieurs mois après le traitement.

5. Spécificité des réactions en fonction des antigènes

En opposant les sérums des animaux d'expérience à différents antigènes, on a pu constater :

a) que l'antigène $T$. brucei réagit fortement avec les sérums homologues et les sérums hétérologues; cet antigène s'est révélé le meilleur pour la pratique de l'immunofluorescence des trypanosomiases animales; b) que l'antigène $T$. congolense donne de moins bonnes réactions, même avec des sérums qui lui sont homologues;

c) que l'antigène $T$. vivax réagit bien avec les sérums homologues mais aussi, de façon égale, avec d'autres sérums;

d) que l'antigène $T$. theileri de culture donne une réaction de fluorescence avec un sérum provenant d'une infection à $T$. brucei, mais à la dilution $1 / 10$.

Nous n'avons pu disposer que d'un sérum de bovin uniquement parasité par $T$. theileri et il n'est positif qu'à $1 / 10$ avec l'antigène homologue.

Il apparaît en tout cas que la méthode d'immunofluorescence indirecte, telle que pratiquée ici, ne permet pas, du fait des réactions croisées, un diagnostic monospécifique des espèces de trypanosomes. Cette constatation, ajoutée à la persistance des anticorps chez un animal traité, limite considérablement l'intérêt de la méthode dans le diagnostic des trypanosomiases animales. Mais qu'en est-il lorsqu'il s'agit de surveillance épizootiologique?

\section{Infections naturelles et surveillance épizootiologique}

De mai 1973 à avril 1975, plusieurs centaines de sérums ont été analysés, provenant de bovins d'origine ou de répartition géographique diverse, pour juger de la valeur de la méthode d'immunofluorescence dans l'étude épizootiologique des trypanosomiases. Conjointement, pour chaque préparation sérologique, des lectures de lames colorées au Giemsa permettent une étude critique des résultats obtenus. Toutes les épreuves ont été faites en utilisant comme antigène $T$. brucei.

\section{Bovins de Koungheul et Kaffrine}

L'enquête relatée ici porte d'une part sur 
des troupeaux d'unités expérimentales suivies par le Centre de Recherches agronomiques de Bambey et situées autour de Koungheul, d'autre part sur des animaux de Kaffrine. Les localités intéressées sont hors de la limite de distribution des glossines et les animaux ne risquent que très faiblement d'être trypanosomés.

Au total, 152 animaux ont été contrôlés par frottis et gouttes épaisses. Aucun n'héberge apparemment de trypanosomes. Par contre, 12 d'entre eux sont parasités soit par Babesia mutans, soit Anaplasma marginale, soit enfin des microtilaires de Setaria.

L'étude de 83 échantillons de sang prélevés sur papier filtre dans les villages des unités expérimentales a donné des résultats négatifs en immunofluorescence avec un antigène $T$. brucei et une dilution sérologique sensiblement égale à $1 / 80$ (disque imprégné de sang de $9 \mathrm{~mm}$ de diamètre, élué dans $1,3 \mathrm{ml}$ de tampon phosphate). Négatifs aussi sont les résultats obtenus avec 36 sérums récoltés à Kaffrine et dilués à $1 / 80$.

Les animaux hébergeant des parasites autres que les trypanosomes font partie des 119 dont le sang a été analysé et on peut dire qu'il n'y a pas de réaction croisée entre les trypanosomes et les autres parasites sanguins trouvés chez ces bovins.

\section{Bovins de Sokone}

Les animaux de cette localité sont soumis à un grand risque de trypanosomiase du fait de la présence de glossines (Glossina palpalis gambiensis et $G$. morsitans submorsitans). La plupart des bovins élevés sont de race Ndama mais on trouve aussi des Diakoré, métis de Zébu et Ndama. Sur 66 bovins, 7 ont une parasitémie apparente sur les lames colorées, (5 T. congolense et $2 T$. vivax), soit 10,6 p. 100 . Par la méthode d'immunofluorescence, 18 réactions sont positives sur les 27 sérums récoltés, soit 66 p. $100: 11$ réagissent à $1 / 80$ et 7 à $1 / 320$. Le calcul de la G.M.R.T. donne 26,5 dans cette observation. Il n'y a pas de réactions croisées avec Babesia begemina et les microfilaires.

\section{Zébus Guzera du Brésil}

Leur état est inconnu : il s'agit de 24 animaux, importés du Brésil et mis en quarantaine à Dakar. Aucun n'a de parasitémie lisible et les hémocuitures pour déceler $T$. theileri sont aussi négatives. A l'immunofluorescence, le sérum de 4 des animaux est à la limite du positif, à la dilution $1 / 80$, et celui des 20 autres est négatif. En considérant les 4 sérums comme positifs à $1 / 40$, on obtient un G.M.R.T. égal à 1,8 . Ces animaux sont vraisemblement indemnes de trypanosomiase.

\section{Zébus du département de Matam}

La surveillance porte sur une région sans glossines. Dans un effectif de 128 Zébus, les lames colorées au Giemsa n'ont révélé de trypanosomes que chez 2 d'entre eux ( $T$. vivax, $T$. theileri). L'immunofluorescence, pratiquée sur 29 sérums et à deux reprises, donne des résultats différents : tous les sérums sont positifs entre $1 / 80$ et $1 / 320$ et la G.M.R.T. est égale à 156,3 . Bien que, dans leur grande majorité, ces animaux n'hébergent pas de trypanosomes, on peut dire qu'il y a eu une forte enzootic dans les semaines ou les mois qui ont précédé les prélèvements. L'enquête nous a révélé que, du fait de la sécheresse, ils ont en effet transhumé dans le sud du pays, infesté de glossines, et qu'au cours de cette transhumance ils ont pu contracter la trypanosomiase. De plus, ces bovins venaient de recevoir un traitement trypanocide dans le cadre d'une " opération de sauvegarde du bétail $»$.

\section{Bovins de l'arrondissement de Maka}

Les animaux des agglomérations visitées sont des croisements de Zébus et Ndama. Les prospections, faites en mars 1974, n'ont permis de déceler que de très rares glossines autour de Maka (G. morsitans submorsitans et $G$. palpalis gambiensis). Sur 209 analyses microscopiques, il n'y en a qu'une seule positive ( $T$. vivax) tandis que sur les 24 sérums rapportés au laboratoire, 12 sont positifs à $1 / 80$ (G.M.R.T. = 89). L'état des animaux est relativement bon et on constate une moindre enzootie de la trypanosomiase par rapport à une situation antérieure. Cela est lié à une très faible infestation en glossines du fait de la sécheresse de 1973.

\section{Zébus de Dahra}

Huit analyses sérologiques sur des Zébus du Centre de Recherches zootechniques de Dahra ont donné des résultats négatifs. Cela confirme l'absence de trypanosomiase dans ce centre, très éloigné des régions à glossines. Il en est de même pour 9 autres analyses faites en février 1975. 
TABL, $N^{\circ} \mathrm{VI-Analyse} \mathrm{de} \mathrm{sërums} \mathrm{rêcoltếs} \mathrm{à} \mathrm{Kolda.}$

\begin{tabular}{|l|c|c|c|c|c|c|c|}
\hline \multicolumn{1}{|c|}{ Villages } & Nég. & $1 / 40$ & $1 / 80$ & $1 / 160$ & $1 / 320$ & N & G.M.R.T. \\
\hline Madına Yéro Bula & 3 & 1 & 3 & 3 & 0 & 10 & 24,6 \\
Saré Guéladio & 9 & 2 & 3 & 3 & 2 & 19 & 12,0 \\
Bantan Kountouyel & 4 & 0 & 0 & 0 & 0 & 04 & - \\
Fafakourou & 1 & 3 & 3 & 1 & 3 & 11 & 69,1 \\
Santankoyel & 3 & 4 & 3 & 2 & 1 & 13 & 29,0 \\
Saré Sara & 2 & 2 & 0 & 0 & 0 & 04 & - \\
Cuiro Bocari & 1 & 1 & 1 & 0 & 0 & 03 & - \\
Dar Salaam & 1 & 1 & 0 & 0 & 0 & 02 & - \\
Mahon Bantanto & 1 & 0 & 0 & 0 & 0 & 01 & - \\
Lingue to & 1 & 0 & 0 & 0 & 0 & 01 & - \\
Madina Lađji & 0 & 0 & 0 & 0 & 1 & 01 & 69 \\
\hline Kalda & 26 & 14 & 13 & 9 & 7 & 69 & - \\
\hline
\end{tabular}

\section{Ndama de Kolda}

Au cours de mai 1974 des enquêtes dans différents troupeaux de l'agglomération de Kolda, portant sur 298 bovins de race Ndama ont donné 34 cas positifs par la méthode standard (T. congolense ou T. vivax), soit 11,4 p. 100 . C'est une incidence non négligeable d'autant plus qu'il s'agit d'animaux de race Ndama. La présence de $T$. theileri n'a été constatée que sur 2 lames mais une étude antérieure par hémoculture nous avait montré une incidence de cette espèce chez plus de 70 p. 100 des bovins de l'agglomération (15). L'analyse de 69 sérums prélevés dans plusieurs villages donne les résultats suivants (toujours avec l'antigène $T$. brucei) :

Le animaux positifs parasitologiquement sont positifs à l'immunofluorescence sauf un, infecté par T. vivax. Beaucoup d'animaux parasitologiquement négatifs sont positifs à l'immunofluorescence et on atteint 62,3 p. 100 de réactions positives. Il est à noter que les titres d'anticorps se rapportent ici à des réaction obtenues avec un conjugué anti-IgM bovine et qu'ils sont sans doute moins élevés que dans des réactions qui utiliseraient un conjugué anti-globutines totales.

\section{DISCUSSION}

De toute évidence, l'interprétation des faits est malaisée avec la méthode indirecte d'immunofluorescence et de nombreuses questions doivent être posées avant toute conclusion. L'acceptation d'un résultat comme positif est,
TABL.N`VII-Récapitulation des résultats des enquêtes

\begin{tabular}{|c|c|c|c|}
\hline Localitê & Glassines & $\begin{array}{l}\text { Trypanosomes } \\
\text { pathogènes }\end{array}$ & GMRT \\
\hline Dahra & néant & néant & 0 \\
\hline Koungheul & néant & néar.t & 0 \\
\hline Kaffrine & nêant & nëant & 0 \\
\hline Brêsil & ne ant & néant & 1,8 \\
\hline Maka & $\begin{array}{l}\text { G.morsitans subm. } \\
\text { G. paipalis gamb. } \\
\text { (Très rares) }\end{array}$ & $\begin{array}{l}\text { T. vivax } \\
\text { (1 fois) }\end{array}$ & $B, 9$ \\
\hline Sokone & $\begin{array}{l}\text { G. morsitana subm. } \\
\text { G. palpalia gamb. }\end{array}$ & $\begin{array}{l}\text { T. congolense } \\
\text { T. vivax }\end{array}$ & 26,5 \\
\hline Kolda & $\begin{array}{l}\text { G.morsitans subm. } \\
\text { G.palpalis gamb. }\end{array}$ & $\begin{array}{l}\text { T. congolense } \\
\text { T. vivax }\end{array}$ & 16,8 \\
\hline Matam & $\begin{array}{c}\text { nêant } \\
\text { (mais transhumance) }\end{array}$ & $\begin{array}{l}\text { T. vivax } \\
(1 \text { fois })\end{array}$ & 156,3 \\
\hline
\end{tabular}

à la limite, lié à quelque subjectivité de l'observateur. Un sérum qui réagit à $1 / 40$ peut certainement être considéré comme positif mais sans doute pas à une dilution plus basse (22). En tout cas, pensons-nous, il y a lieu, pour un même animal, de faire une gamme de dilutions permettant de procéder à des évaluations critiques dans l'échelle de fluorescence adoptée avec un même antigène.

La méthode quoique sensible, ne permet pas de faire un diagnostic de trypanosomiase n'ayant évolué que quelques semaines. Cette opinion est partagée par MWAMBU et OMASET, (9) qui pensent que les anticorps ne sont décelables qu'à la phase clinique de la maladie; de même par ASHKAR et OCHILO, (3) et WILSON, (22). 
Cette sensibilité est plus grande dans le système antigène-anticorps homologues de $T$. brucei qu'elle ne l'est dans les réactions hétérologues. L'antigène $T$. congolense nous a donné de mauvais résultats même avec des sérums homologues, mais il faut préciser que la souche utilisée a été passée plusieurs centaines de fois sur souris. En utilisant comme antigène T. brucei dans les épreuves de diagnostic et d'épizootiologie, il ressort des résultats que la méthode d'immunofluorescence n'a dans les trypanosomiases qu'une spécificité de groupe (nous préférons dire : réactivité générique, en l'apposant à une réactivité spécifique). En effet, on distingue bien cette maladie des autres affections parasitaires du sang: des bovins sans trypanosomes mais portant Babesia bigemina, B. mutans, Anaplasma marginale ou des microfilaires de Setaria $s p$ n'ont, d'après nos observations, donné aucune réaction positive avec un antigène Trypanosoma. Par contre, au niveau spécifique, il y a des réactions croisées et celles-ci ne sont pas neutralisées par la contre coloration au Bleu d'Evans. Cela confirme de nombreuses observations : WAIN et BURRIDGE, (17) notent des réactions croisées entre $T$. vivax et $T$. congolense; ZWART et al., (24) entre T. brucei, $T$. congolense et $T$. vivax, mais ils constatent cependant à haute dilution des différences importantes entre réactions homologues et réactions hétérologues. Enfin WILSON et al., (23) trouvent, comme nous, des réactions croisées entre souches pathogènes (T. congolense, $T$. brucei et $T$. vivax) et souches non pathogènes ( $T$. theileri) bien que, dans ce dernier cas, le titre des anticorps réagissant soit très faible (1/20 et même pas $1 / 40)$. Ce fait est important, car une réaction positive à une dilution plus élevée avec un antigène d'espèce pathogène peut être attribuée à une maladie présente ou passée. Mais il restera difficile de déterminer l'espèce en cause. Cette constatation d'une limite dans le diagnotic monospécifique a aussi été faite par Van MEIRVENNE, (16), ainsi que MEHLITZ et DEINDL, (7) et enfin POLITZAR, (10). Toutefois, SCHINDLER (13), pense que la méthode pourrait conduire à un diagnostic monospécifique des espèces du fait que, selon ses observations, les réactions croisées sont faibles. Il est certain qu'on pourrait, avec plusieurs dilutions éprouvées contre plusieurs antigènes, présumer le trypanosome en cause, mais alors la méthode cesse d'avoir un intérêt pratique pour un diagnostic et une surveillance épizootiologi- que rapides chez les animaux. Toutefois, sa valeur comme méthode de recherches pour caractériser les anticorps au cours des trypanosomiases est indéniable, comme l'ont montré entre autres les expériences de WILSON, (21) et celles de MONIER et al. (8).

Un autre inconvénient lié à la méthode est l'impossibilité de distinguer un animal réellement trypanosomé d'un autre qui l'a été mais a reçu une drogue trypanocide dans un passé récent. Beaucoup insistent sur ce fait, entre autres WIESENHUTTER, (19); SEYDI (14).

A ces restrictions près, la pratique de l'immunofluorescence indirecte garde un intérêt dans les études d'épizootiologie. WIESSENHUTTER, (20) pratiquant des enquêtes en Tanzanie sur 1330 Zébus en zone infestée de glossines et 1170 autres dans une zone indemne arrive à des résultats comparables à ceux de la méthode classique. De même ASHKAR et OCHILO (3) qui ont pratiqué au Kénya des des analyses sur 411 bovins à l'aide de sérums et de prélèvements sur papier filtre trouvent des réactions positives dans 85 à 89 p. 100 des cas d'infection décelés parasitologiquement. Mais ces auteurs précisent que le diagnostic individuel est imprécis.

\section{CONCLUSIONS}

Dans le diagnostic et l'étude épizootiologique des trypanosomiases animales par la méthode indirecte d'immunofluorescence, les incertitudes l'emportent sur les preuves irréfutables :

- si la méthode est très sensible pour déceler la maladie à la phase clinique, elle ne permet pas cependant de dépister les infections précoses même lorsqu'il y a parasitémie;

- de plus elle conduit à déceler plus de cas positifs qu'il n'y a de bovins réellement trypanosomés, surtout lorsque les animaux sont soumis à des traitements trypanocides;

- la méthode n'a principalement qu'une réactivité générique permettant de distinguer les trypanosomiases des autres affections parasitaires; la réactivité spécifique pour distinguer les différentes espèces de trypanosomes existe cependant, mais il n'est pas pratique de faire de nombreuses réactions pour, en définitive, n'aboutir qu'à des résultats exprimés avec probabilité. 
De plus, il est souvent malaisé de faire des saignées dans des troupeaux et de conserver des sérums au cours des tournées.

Un des avantages de la méthode est qu'on peut assez rapidement faire un grand nombre de réactions en économisant les lames et les réactifs. Cependant, toutes proportions gardées, elle est onéreuse du fait des réactifs biologiques et des lampes à vapeur de Mercure qui sont d'un prix élevé pour une durée d'utilisation relativement courte.

Nous conclurons en disant que l'immunofluorescence indirecte a surtout un intérêt dans les études d'épizootiologie ou les recherches sur les anticorps; sa valeur pratique en matière de diagnostic est plus limitée car les réactions positives laissent des incertitudes.

\title{
SUMMARY
}

\author{
Value of the indirect fluorescence antibody test in the \\ diagnosis and the epizootiological survey on bovine \\ trypanosomiases
}

\begin{abstract}
The present paper reviews the results obtained in using the indirect fluorescent antibody test in diagnosis and epizootiological survey of bovine trypanosomiases in Senegal and sums up several critical considerations about the value of this method. The IFT is a quite sensitive method but it fails to detect early cases of Trypanosomiasis. Besides this fact, it leads to more positive cases than do exist really infected animals, mainly when cattle are periodically treated. The IFT gives good generic reactivity allowing distinction between trypanosomiases and other blood parasitic diseases. However at a specific reactivity level it appears that cross reaction between different Trypanosome species limit the value of the method in practical diagnosis purposes. Therefore the IFT seems to be of interest only in epizootiological survey on trypanosomiases and in research on antibodies related to the diseases.
\end{abstract}

\section{RESUMEN \\ Valor del método de inmunofluorescencia indirecta para el diagnóstico de las tripanosomiasis de los bovinos y su estudio epizootiologico}

\begin{abstract}
El autor pasa revista de los resultados obtenidos por el método de inmunofluorescencia indirecta utilizada para el diagnóstico y el estudio epizootiologico de tripanosomiasis de los bovinos en Senegal y discute de modo critico el valor del método. Este es muy sénsible pero no permite rastrear las infecciones precoces. En cambio, demuestra más casos positivos de lo que hay animales atacados por la tripanosomiasis, sobretodo cuando se tratan periodicamente dichos animales.

El método tiene una buena reactividad genérica permitiendo distinguir las tripanosomiasis de otras enfermedades parastarias de la sangre. Al nivel de la reactividad específica, se comproban reacciones cruzadas entre las diferentes especies de tripanosomas, lo que limita el interes práctico en un objeto de diagnóstico. Sin embargo, se puede utilizar el método indirecto de inmunofluorescencia para los estudios epizootiologicos y las búsquedas sobre los anticuerpos en la tripanosomiasis.
\end{abstract}

\section{BIBLIOGRAPHIE}

1. AMBROISE-THOMAS (P.). Etude séro-immunologique sur dix parasitoses par les techniques d'immunofluorescence. Lyon, Institut de Médecine et d'Hygiène tropicales, $1969,645 \mathrm{p}$.

2. AMBROISE-THOMAS (P.). Intérêt de l'immunofluorescence dans le diagnostic, le contrôle postthérapeutique et la surveillance épidémiologique des parasitoses. J. Parasit., 1970, 56 (4) : 4-5.

3. ASHKAR (T.) et OCHILO (M.). The application of the indirect fluorescent antibody test to samples of sera and dried blood from cattle in Lam- bue Valley, South Nyanza, Kenya. Bull. Wld. Hlth. Org., 1972, 47 (6): 769-772.

4. COONS (A. H.) et CREECH (H. J.). Immunological properties of an antibody containing a fluorescent group. Proc. Soc. exp. Biol., New York, 1941, 47: 200-202.

5. CUNNINGHAM (P.M.), WILSON (A.J.) et KIMBER (C.D.). Modification of the indirect fluorescent antibody test as applied to bovine trypanosomiasis. E. Afr. Trypanosomiasis Res. 
Org, Tororo, Uganda, Annual Report, 1966, pp. 20-32.

6. FIFE (E. H.) et MUSCHEL (L. H.). Fluorescent antibody technique for serodiagnosis of Trypanosoma cruzi infection. Proc. Soc. exp. Biol. Med., 1959, 101 : 540-543.

7. MEHLITZ (D.) et DEINDL (G.). Serological studies on cattle experimentally infected with african trypanosomes. Preliminary communication. Zeitschr. Tropenmed. Parasit., 1973, 23 (4) : 411-417.

8. MONIER (J. C.), THIVOLET (J.) et Collab. Signification et valeur diagnostique des différents types d'anticorps anti-nucléaires mis en évidence par immunofluorescence sur frottis de sang de souris trypanosomées. Lyon méd., 1970, 223 (20): 1007-1018.

9. MWAMBU (P. M.) et OMASET (P.). Evaluation of the indirect fluorescent antibody test as used in the diagnosis of bovine trypanosomiasis. $E$. Afr. Trypanosomiasis Res. Org., Tororo, Uganda, Annual Report, 1967, pp. 23-24.

10. POLITZAR (H.). Serological studies on cattle experimentally infected with several species of african trypanosomes. $Z$. Tropenmed. Parasit., $1974,25(1): 22-27$.

11. SADUN (E. H.) et DUXBURY (R. E.). Fluorescent antibody reaction in Trypanosoma rhodesiense and $T$. gambiense in experimental animals. J. Parasit., 1963, 49: 380-384.

12. SADUN (E. H.), DUXBURY (R. E.), WILLIAMS (J.S.) et ANDERSON (R. I.). Fluorescent antibody test for the serodiagnosis of african and american trypanosomiasis in man. J. Parasit., 1963, $49: 385-388$.

13. SCHINDLER (R.). Untersuchungen über die Brauchbarkeit serologischer Verfahrer für die diagnose der Rinder Trypanosomiasis in Afrika. Z. Tropenmed. Parasit., 1972, 23 (1) : 78-88.

14. SEYDI (M.). Contribution à l'étude du diagnostic expérimental des trypanosomoses bovines par immunofluorescence indirecte au Sénégal. Thèse doct. vét. Dakar, 1974, no 1, 99 p.

15. TOURE (S. M.). Description complémentaire de
Trypanosoma theileri Laveran, 1902. Mention particulière de formes observées en Casamance. Rev. Elev. Méd. vét. Pays trop., 1968, 21 (3): 365-373.

16. VAN MEIRVENNE (N.), MOORS (A.) et JANSSENS (P. G.). Serological studies on animals experimentally infected by african trypanosomes. Trans. r. Soc. trop. Med. Hyg., 1972, 66 (2) : 333334.

17. WAIN (E. B.), BURRIDGE (M. J.) et Collab. Incidence of bovine trypanosomiasis in South Bugosa. E. Afr. Trypanosomiasis Res. Org., Tororo, Uganda, Annual Report, 1966, pp. 59-60.

18. WERY (M.), WERY-PASKOFF (S.) et VAN WETTERE (P.). The diagnosis of human african trypanosomiasis (Trypanosoma gambiense) by the use of fluorescent antibody test. Ann. Soc. belge Méd. trop., 1970, 50 (5) : 613-634.

19. WIESENHUTTER (E.). An evaluation of the indirect fluorescent antibody technique in bovine trypanosomiasis after chemoprophylaxis, $Z$. tropenmed. Parasit., 1969, 20 (2): 131-136.

20. WIESENHUTTER (E.). Comparative studies on the value of the indirect florescent antibody test as an aid to diagnosis of bovine trypanosomiasis in Africa. Z. tropenmed. Parasit., 1973, 24 (1) : 60-66.

21. WILSON (A. J.). Pattern of common antibody in bovine trypanosomiasis. Loc. cit. 1967, p. 18.

22. WILSON (A. J.). Value of the indirect fluorescent antibody tests as a serological aid to diagnosis of glossina-transmitted trypanosomiasis. Trop. anim. Hith. Prod., 1969, 1 (2): 89-95.

23. WILSON (A. J.), CUNNINGHAM (P.M.) et KIMBER (C. D.). The indirect fluorescent antibody test applied to bovine trypanosomiasis. $E$. Afr. Tryp. Res. Org., Tororo, Uganda, Annual Report, 1966, pp. 28-29.

24. ZWART (D.), FERIE (N. M) et Collab. A comparison of methods for diagnosis of trypanosomiasis in East African domestic ruminants. Trop. anim. Hlth. Prod., 1973, 5 (2) : 79-86. 